\title{
FATORES DE RISCO PARA INFECÇÃO DO SÍTIO CIRÚRGICO EM CIRURGIAS POTENCIALMENTE CONTAMINADAS
}

\author{
Tatiana Martins ${ }^{1}$, Lúcia Nazareth Amante², Janeisa Franck Virtuoso ${ }^{3}$, Bruna Telemberg Sell', Jeane Silvestri \\ Wechi ${ }^{5}$, Carla Vieira Amante Senna ${ }^{6}$
}

${ }^{1}$ Mestre em Enfermagem. Enfermeira da Estratégia de Saúde da Família do Município de Biguaçu. Biguaçu, Santa Catarina, Brasil. E-mail: tatiana_martins15@hotmail.com

${ }^{2}$ Doutora em Enfermagem. Professora do Departamento de Enfermagem da Universidade Federal de Santa Catarina (UFSC). Florianópolis, Santa Catarina, Brasil. E-mail: lucidamente@gmail.com

${ }^{3}$ Doutora em Fisioterapia. Professora do Departamento de Fisioterapia da UFSC. Araranguá. Santa Catarina, Brasil. E-mail: janeisav@yahoo.com.br

${ }^{4}$ Mestre em Enfermagem. Enfermeira da Secretaria Municipal de Saúde do Município de São José e do Hospital Governador Celso Ramos. Florianópolis, Santa Catarina, Brasil. E-mail: sellbruna@hotmail.com

${ }^{5}$ Mestre Profissional em Gestão do Cuidado em Enfermagem. Enfermeira do Hospital Universitário da UFSC. Florianópolis, Santa Catarina, Brasil. E-mail: jeanewechi@hotmail.com

${ }^{6}$ Mestre em Enfermagem. Enfermeira do Hospital Infantil Joana de Gusmão. Florianópolis, Santa Catarina, Brasil. E-mail: cvsenna@ hotmail.com

\section{RESUMO}

Objetivo: associar os fatores de risco do período pós-operatório de cirurgias potencialmente contaminadas, com a ocorrência da infecção do sítio cirúrgico no período pós-operatório hospitalar e domiciliar.

Método: período de coleta de dados foi de fevereiro a junho de 2015, com 84 pacientes acompanhados nos três primeiros dias de pósoperatório e sete dias após a alta hospitalar, através de questionários elaborados para avaliação do sítio cirúrgico, das condições clínicas do paciente e da análise de prontuário. Para verificação dos fatores de risco influenciáveis e classificação das infecções do sítio cirúrgico, utilizou-se um questionário com variáveis que foram analisadas de forma descritiva através da frequência simples, porcentagem e medidas de posição e dispersão. Para a associação e nível de significância dos fatores de risco foram realizados testes estatísticos.

Resultados: nenhum dos pacientes entrevistados desenvolveu infecção profunda do sítio cirúrgico ou de órgão/espaço, sendo que sete desenvolveram infecção do sítio cirúrgico superficial no pós-operatório hospitalar e 41 em domicílio. Os fatores de risco analisados não foram significativos para o desenvolvimento das infecções.

Conclusões: recomenda-se que as instituições implantem um serviço de vigilância e acompanhamento pós-alta, a fim de que se melhore a confiabilidade das taxas de incidência das infecções do sítio cirúrgico; melhorar a qualidade dos cuidados com os pacientes; compreensão epidemiológica e identificação dos fatores de risco. Além disso, propõe-se facilitar o processo de referência e contra referência através de capacitações da Estratégia de Saúde da Família para identificação destes fatores de risco, atuando em rede.

DESCRITORES: Segurança do paciente. Assistência perioperatória. Infecção da ferida operatória. Fatores de risco.

\section{RISK FACTORS FOR SURGICAL SITE INFECTIONS IN POTENTIALLY CONTAMINATED SURGERIES}

\begin{abstract}
Objective: to associate the risk factors of the postoperative period of potentially contaminated surgeries, with the occurrence of surgical site infections in the postoperative period in the hospital and home setting.

Method: the data collection period was from February to June 2015, 84 patients were followed up on the first three post-operative days and seven days after hospital discharge, the collection was performed through questionnaires designed to evaluate the surgical site, the clinical conditions of the patient and chart analysis. In order to verify the influencing risk factors and classifications of surgical site infections, a questionnaire was used with variables that were analyzed in a descriptive manner through simple frequency, percentage, and position and dispersion measurements. Statistical tests were performed for the association and significance level of the risk factors.

Results: none of the patients interviewed developed a deep infection of the surgical or organ / space site, seven developed superficial surgical site infections in the post-operative period in hospital and 41 developed a surgical site infection at home. The analyzed risk factors were not significant for the development of infections.

Conclusions: it is recommended that institutions implement a post discharge monitoring and follow-up service in order to improve the reliability of the incidence rates of surgical site infections; improving the quality of patient care; epidemiological understanding and identification of risk factors. In addition, it proposes to facilitate the referral and counter referral process through training in the Family Health Strategy in order to identify these risk factors, and by acting in a network.
\end{abstract}

DESCRIPTORS: Patient safety. Perioperative care. Surgical Site Infection. Risk factors. 


\title{
FACTORES DE RIESGO PARA LA INFECCIÓN DEL SITIO QUIRÚRGICO EN CIRUGIAS POTENCIALMENTE CONTAMINADAS
}

\begin{abstract}
RESUMEN
Objetivo: asociar los factores de riesgo del período postoperatorio de cirugías potencialmente contaminadas, con la ocurrencia de la infección del sitio quirúrgico en el período postoperatorio hospitalario y domiciliar.

Método: período de recolección de datos fue de febrero a junio de 2015, con 84 pacientes acompañados en los tres primeros días de postoperatorio y siete días después del alta hospitalaria, a través de cuestionarios elaborados para evaluación del sitio quirúrgico, de las condiciones clínicas del paciente y del análisis de prontuario. Para la verificación de los factores de riesgo influenciables y clasificación de las infecciones del sitio quirúrgico, se utilizó un cuestionario con variables que fueron analizadas de forma descriptiva a través de la frecuencia simple, porcentaje y medidas de posición y dispersión. Para la asociación y el nivel de significancia de los factores de riesgo se realizaron pruebas estadísticas.
\end{abstract}

Resultados: ninguno de los pacientes entrevistados desarrolló una infección profunda del sitio quirúrgico o de órgano/espacio, siendo que siete desarrollaron infección del sitio quirúrgico superficial en el postoperatorio hospitalario y 41 en domicilio. Los factores de riesgo analizados no fueron significativos para el desarrollo de las infecciones.

Conclusiones: se recomienda que las instituciones implantes un servicio de vigilancia y seguimiento post-alta, a fin de que se mejore la confiabilidad de las tasas de incidencia de las infecciones del sitio quirúrgico; mejorar la calidad del cuidado de los pacientes; la comprensión epidemiológica y la identificación de los factores de riesgo. Además, se propone facilitar el proceso de referencia y contra referencia a través de capacitaciones de la Estrategia de Salud de la Familia para identificar estos factores de riesgo, actuando en red.

DESCRIPTORES: Seguridad del paciente. Asistencia perioperatoria. Infección de la herida operatoria. Factores de riesgo.

\section{INTRODUÇÃO}

As infecções do Sitio Cirúrgico (ISC) apresentam riscos à segurança do paciente nos serviços de saúde do Brasil, tendo grande importância nas Infecções relacionadas à assistência à saúde. As ISC acometem pacientes submetidos a procedimentos cirúrgicos, com ou sem colocação de implantes, internados ou em nível ambulatorial, acometendo tecido subcutâneo, moles profundos (fáscia e músculo), órgão e cavidades com incisão, sendo caracterizadas como aquelas que ocorrem até o $30^{\circ}$ dia de pós-operatório. ${ }^{1-4}$

A fonte mais comum de microrganismos é a flora endógena do paciente. Estima-se que no pós-operatório imediato, o sítio cirúrgico esteja protegido da contaminação exógena. Estas fontes exógenas devem ser consideradas durante o procedimento cirúrgico, através de uma técnica asséptica precisa, sendo conservada no intuito de prevenir a contaminação. ${ }^{1,3,5}$

Em acordo com a Centers for Disease Control and Prevention (CDC), o diagnóstico epidemiológico da ISC é feito através da análise de alguns parâmetros: aparecimento da infecção em até 30 dias pós-operatório, ou em casos de implantes de próteses, em até um ano..$^{6-8}$

A relação das ISC com a possível contaminação da cirurgia já foi ratificada em alguns estudos. ${ }^{9-15}$ As cirurgias potencialmente contaminadas, classificadas em classe II quanto ao seu potencial de contaminação, são realizadas em tecidos colonizados por flora microbiana pouco numerosa ou nos tecidos colonizados ausentes dos processos de infecção e inflamação. ${ }^{1,16}$
A contaminação do sítio cirúrgico pode acontecer em qualquer momento do período perioperatório, sendo decorrente dos fatores de risco relacionados ao paciente, ao procedimento cirúrgico e ao ambiente hospitalar desde o período pré-operatório até o pós-operatório domiciliar. Alguns dos fatores associados a estes períodos podem ser: tempo de cirurgia, local de cirurgia, tipo de cirurgia realizada, tempo de internação hospitalar, fatores relacionados à microrganismos, fatores relacionados ao paciente, entre outros. Sendo que a identificação e o controle destes fatores de riscos devem ocorrer desde o primeiro contato do paciente com a instituição, por meio de coleta de informações relacionadas ao plano de cuidados. ${ }^{16}$

O serviço de controle de infecção hospitalar, do hospital em que foi realizado esta pesquisa, registrou no ano de 2013 que foram realizados 2259 procedimentos cirúrgicos, dos quais 1248 $(55,25 \%)$ foram em cirurgias potencialmente contaminadas. A taxa de IH foi de $82(6,57 \%)$ sendo que $35(2,80 \%)$ desenvolveram ISC. Diante desta realidade, fez-se um levantamento dos fatores de risco pós-operatório hospitalar, sobre o surgimento de ISC durante o mesmo período, bem como no pós-operatório domiciliar. O local de realização da pesquisa ocorreu nas unidades de internação cirúrgicas do hospital escolha. ${ }^{15}$

É fundamental que o enfermeiro compreenda os fatores de risco que predispõem a incidência de ISC no pós-operatório para promover ações e medidas que reduzam a ocorrência destas, colaborando na melhoria da qualidade da assistência e na inserção do sistema de vigilância sobre o controle 
das infecções e ao acesso as informações que sirvam de base para a prevenção. ${ }^{16}$

A prestação de cuidados e assistência segura de enfermagem ao paciente cirúrgico, destacando o período pós-operatório é indispensável para a identificação dos fatores de risco. Buscando relacionar estes fatores com as ISC, apresenta-se a seguinte pergunta de pesquisa: existe correlação dos fatores de risco do período pós-operatório hospitalar com as ISC no período pós-operatório hospitalar e domiciliar de cirurgias potencialmente contaminadas? Desta forma, objetivou-se associar os fatores de risco do período pós-operatório de cirurgias potencialmente contaminadas, com a ocorrência da infecção do sítio cirúrgico no período pós-operatório hospitalar e domiciliar.

\section{MÉTODO}

Trata-se de uma pesquisa transversal, descritiva, com abordagem quantitativa, realizada em duas unidades de internação cirúrgica de um Hospital de ensino da Região Sul do Brasil, no período de 12 de fevereiro de 2015 a 30 de junho de 2015, tendo em vista a aprovação do projeto de pesquisa pela Instituição de escolha. O hospital de escolha possui 274 leitos hospitalares, sendo que uma das unidades de internação cirúrgica possuem 30 leitos distribuídos em 12 quartos, onde internam pacientes em períodos de pré e pós-operatórios das seguintes especialidades cirúrgicas: cabeça e pescoço, gastrointestinal e vias biliares, torácica, otorrinolaringologia, bucomaxilofacial, transplante hepático e neurocirurgia. Já a segunda unidade também possui 30 leitos, distribuídos em 12 quartos, que atendem as especialidades de urologia, proctologia, cirurgia plástica e vascular. ${ }^{15}$

Os critérios de inclusão foram: participantes maiores de 18 anos de idade ou iguais; de ambos os sexos; em pós-operatório de cirurgias potencialmente contaminadas eletivas; que possuírem o número de telefone disponível para contato pós-alta hospitalar e que puderam manter um acompanhamento durante o pós-operatório hospitalar, até o dia da sua alta. Excluíram-se os participantes submetidos a cirurgias prévias já contaminadas; os que já haviam sido inseridos na amostra em algum momento, reinternados e aqueles com qualquer tipo de infecção sistêmica confirmada. Para representatividade da amostra, foi realizado um cálculo pelo Sistema de Ensino-Aprendizagem de Estatística na Web (SEstatNet) composto por módulos de interação com funcionalidades específicas, quais sejam: pesquisa, base de dados, análise de dados, distribuições probabilísticas e auto avaliação. $\mathrm{O}$ módulo pesquisa auxiliou na determinação do tamanho da amostra e da maneira de se selecionar os elementos que a compuseram em que foram recrutados 84 pacientes. ${ }^{17}$

Para a realização do cálculo da amostra foi necessário solicitar à instituição os dados referentes aos pacientes e procedimentos realizados nas unidades referência do estudo. Neste caso, para se calcular o tamanho mínimo da amostra, o n do estudo foram os pacientes submetidos a procedimentos cirúrgicos classificados como cirurgias potencialmente contaminadas, totalizando $1248 .{ }^{15}$

Para a assimilação dos fatores de risco (variáveis independentes) relevantes durante o período pós-operatório com a presença ISC no pós-operatório hospitalar e domiciliar, foi utilizado um roteiro de entrevista com perguntas realizadas oralmente e por telefone, aplicado durante o período pós-operatório hospitalar que contemplaram categorias influenciáveis ao desenvolvimento da ISC. Este questionário teve uma testagem através de um teste piloto realizado 20 dias anteriores a coleta de dados com 15 participantes, em um período de 10 dias. Para a identificação das ISC (variáveis dependentes) avaliou-se as características dos curativos em: seco e limpo; presença ou não de dor, hiperemia, calor, edema e deiscência. Para a classificação das ISC em incisionais superficiais, foi avaliado a presença de dor, rubor, calor, edema, febre, deiscência e exsudato purulento.

Os pacientes foram acompanhados durante a cirurgia até o período de alta hospitalar e após sete dias da mesma, foi feito contato telefônico com todos os participantes, no intuito de acompanhar as intercorrências da evolução cicatricial do sítio cirúrgico. O sujeito respondeu algumas perguntas relacionadas ao processo de cicatrização da incisão cirúrgica e da condição clínica. O questionário foi elaborado com questões fechadas em que a pesquisadora assinalava as respostas conforme a resposta do paciente; avaliação do sítio cirúrgico e condições clínicas do paciente; bem como informações extraídas de prontuário.

Os dados foram exportados e analisados no pacote estatístico SPSS ${ }^{\circledR}$ - Statistical Package for Social Sciences (versão 22.0). As variáveis dependentes e independentes foram analisadas de forma descritiva através da frequência simples e de porcentagem e medidas de posição e dispersão. Na associação foi utilizado o teste Qui Quadrado ( $\chi 2)$, para análise bivariada dos fatores de risco com a presença/ ausência de ISC. 
A Regressão Logística Binária foi empregada para relacionar a presença/ausência de ISC no pós-operatório hospitalar e domiciliar com os fatores de risco do período pós-operatório. No modelo de regressão, foram inseridas e consideradas as variáveis com o $p \leq 0,200$ e aceitação do nível de significância de $5 \%$. Para certificação da influência o intervalo de EXP (B) - OR foi igual a 1.

As variáveis associadas ao desenvolvimento da ISC no pós-operatório hospitalar e domiciliar foram: colecistectomia como tipo de cirurgia realizada; diagnóstico pós-operatório de colecistite aguda e colelitíase; tempo de internação até a alta hospitalar e uso de oxigenioterapia. Já as categorias que não se associaram com a ocorrência de ISC nestes períodos foram: gastroplastia como cirurgia realizada; diagnóstico de obesidade; pós-operatório condicional à Unidade de Terapia Intensiva (UTI); jejum e características do curativo do sítio cirúrgico, ainda que o valor de $p>0,200$ nestes fatores.

A pesquisa foi aprovada pelo Comitê de Ética e Pesquisa com Seres Humanos (CEPSH) obtendo o Certificado de Apresentação para Apreciação Ética (CAAE) de número 39866414.1.0000.0115.

\section{RESULTADOS}

As variáveis foram analisadas descritivamente por meio de frequência simples e porcentagens (variáveis categóricas) e medidas de posição e dispersão (variáveis numéricas).

Para a associação entre variáveis categóricas utilizou-se o teste de Qui-Quadrado ( $\chi 2$ ) ou Exato de Fisher, quando necessário. A comparação entre dois grupos, com variáveis numéricas foi realizada através do teste t para amostras independentes ou teste de Mann-Whitney, conforme a distribuição dos dados. Para verificar a distribuição dos dados (normalidade) foi realizado o teste de Kolmogorov-Smirnov. Para o cálculo do valor de ODDS RATIO (OR) e Intervalos de Confiança (IC) foi utilizada a
Regressão Logística Binária entre a presença de ISC e os fatores de risco no pós-operatório hospitalar e domiciliar.

Foram inseridas no modelo de regressão somente as variáveis com $\mathrm{p} \leq 0,200$ na comparação ou associação com a presença de ISC. Adotou-se um nível de significância de $5 \%(p=0,05)$ em que o limite baseado afirma se o desvio é proveniente do acaso ou não. Para a confirmação de influência dos fatores de risco na ocorrência de ISC, o intervalo de EXP (B) - OR foi de 1. Interpretou-se para os valores abaixo de 1 como fator de proteção ao desenvolvimento de ISC e para acima de 1 , os fatores de risco. Exemplo: OR=0,50, interpreta-se como $50 \%$ menos chance de desenvolver ISC com relação a categoria de referência. $\mathrm{E} \mathrm{OR}=5,0$ cinco vezes mais chances de influenciar no surgimento da ISC baseado na categoria de origem. Para as variáveis que não foram coletadas considerou-se a relevância significativa.

Dos 84 participantes, o desenvolvimento da ISC no pós-operatório institucional ocorreu em sete $(8,3 \%)$ pacientes que passaram por cirurgias potencialmente contaminadas. Para a combinação dos fatores de risco do pós-operatório hospitalar com a presença/ausência de ISC no mesmo período, utilizou-se a análise bivariada, através do teste $\chi 2$, conforme tabela 1, considerando nível de associação mais evidente, a categoria diagnóstico de colelitíase obteve $\chi^{2}=3,424$ e $p=0,064$.

Para a análise do tempo de internação até a alta hospitalar, utilizou-se o teste $U$ de Mann Whitney com $U=155,000$ e $p=0,063$. Realizou-se o teste, pois não houve categoria de referência, tampouco homogeneidade nas variâncias com a presença/ ausência de ISC no pós-operatório hospitalar. A média do tempo de internação dos pacientes que tiveram ISC foi de 13,71 dias ( $D P=8,015)$. A incidência de ISC no pós-operatório hospitalar foi em três $(42,9 \%)$ participantes diagnosticados com colecistite aguda. 
Tabela 1 - Análise bivariada entre os fatores de risco modificáveis do período pós-operatório com a presença/ausência de infecções do sitio cirúrgico no período pós-operatório hospitalar. Florianópolis, SC, Brasil, 2015

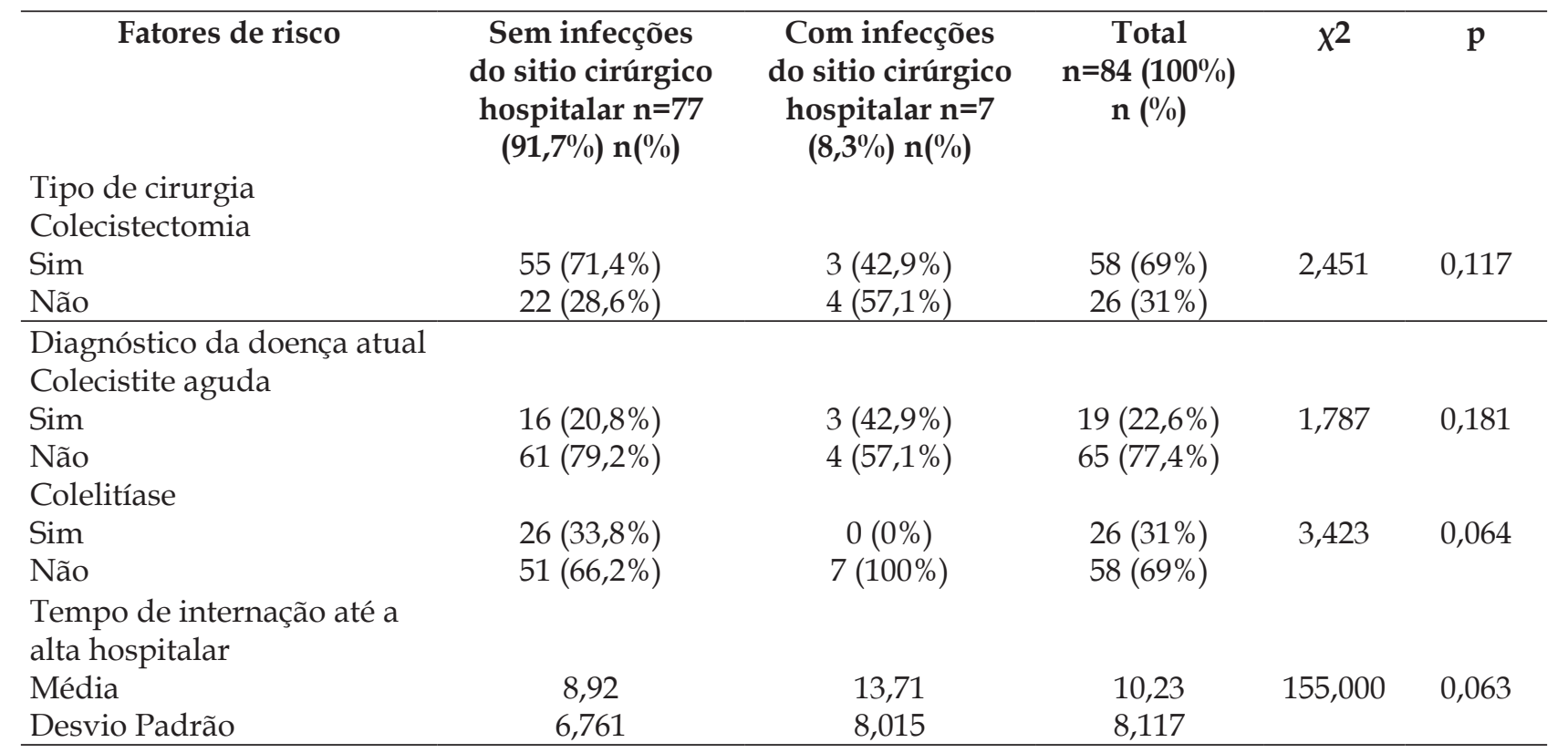

X2: Qui Quadrado; $p$ : nível de significância.

Pela tabela 2 foi possível verificar que a prevalência de ISC no pós-operatório em domicílio foi maior, equivalendo a $41(48,8 \%)$ casos. Através do teste $\chi^{2}$ foi constatado que a associação dos fatores do pós-operatório hospitalar com a presença/ ausência de ISC no domicílio teve maior influência na variável oxigenioterapia $\left(\chi^{2}=3,176\right.$ e $\left.p=0,07\right)$.
Dos pacientes que desenvolveram a ISC domiciliar, $25(61 \%)$ foram submetidos ao procedimento de colecistectomia. Utilizou-se o teste $U$ de Mann Whitney para avaliação da categoria, tempo de internação hospitalar com apresentação de $U=724,500$ e $p=0,159$, sendo a média de internação dos que tiveram a ISC domiciliar de 8,51 dias $(\mathrm{DP}=6,81)$.

Tabela 2 - Análise bivariada dos fatores de risco modificáveis do período pós-operatório com a presença/ ausência da infecções do sitio cirúrgico no pós-operatório domiciliar. Florianópolis, SC, Brasil, 2015

\begin{tabular}{|c|c|c|c|c|c|}
\hline Fatores de risco & $\begin{array}{c}\text { Sem infecções } \\
\text { do sitio cirúrgico } \\
\text { domiciliar } n=43 \\
(51,2 \%) n(\%)\end{array}$ & $\begin{array}{l}\text { Com infecções } \\
\text { do sitio cirúrgico } \\
\text { domiciliar } n=41 \\
(48,8 \%) n(\%)\end{array}$ & $\begin{array}{c}\text { Total } \\
\mathrm{n}=84(100 \%) \\
\mathrm{n}(\%)\end{array}$ & $x^{2}$ & $p$ \\
\hline $\begin{array}{l}\text { Tipo de cirurgia } \\
\text { Colecistectomia } \\
\text { Sim } \\
\text { Não }\end{array}$ & $\begin{array}{l}33(76,7 \%) \\
10(23,3 \%)\end{array}$ & $\begin{array}{l}25(61 \%) \\
16(39 \%)\end{array}$ & $\begin{array}{l}58(69 \%) \\
26(31 \%)\end{array}$ & 2,442 & 0,118 \\
\hline $\begin{array}{l}\text { Oxigenioterapia } \\
\text { Sim } \\
\text { Não }\end{array}$ & $\begin{array}{c}9(20,9 \%) \\
34(79,1 \%)\end{array}$ & $\begin{array}{c}3(7,3 \%) \\
38(92,7 \%)\end{array}$ & $\begin{array}{l}12(14,3 \%) \\
72(85,7 \%)\end{array}$ & 3,176 & 0,075 \\
\hline $\begin{array}{l}\text { Tempo de internação até a } \\
\text { alta hospitalar } \\
\text { Média } \\
\text { Desvio Padrão }\end{array}$ & $\begin{array}{c}10,09 \\
7,07\end{array}$ & $\begin{array}{l}8,51 \\
6,81\end{array}$ & 18,68 & 724,500 & 0,159 \\
\hline
\end{tabular}

X²: Qui Quadrado; p: nível de significância 
Em acordo com a tabela 3, tanto na análise bruta, quanto na ajustada não foi identificado nenhuma variável com nível de significância para o surgimento da ISC no período pós-operatório hospitalar.

Tabela 3 - Análise de regressão logística binária dos fatores de risco do período pós-operatório na presença de infecções do sitio cirúrgico no pós-operatório hospitalar. Florianópolis, SC, Brasil, 2015

\begin{tabular}{|c|c|c|c|c|}
\hline \multirow[t]{2}{*}{ Fatores de risco } & \multicolumn{2}{|c|}{ Análise bruta } & \multicolumn{2}{|c|}{ Análise ajustada } \\
\hline & $\mathbf{R C}^{*}$ & IC 95\%† & $\mathbf{R C}^{*}$ & IC 95\%† \\
\hline \multicolumn{5}{|l|}{ Tipo de cirurgia } \\
\hline \multicolumn{5}{|l|}{ Colecistectomia } \\
\hline Sim & 0,30 & $0,06-1,45$ & 0,00 & $0,00-0,00$ \\
\hline Não & 1,00 & & 1,00 & \\
\hline \multicolumn{5}{|c|}{ Diagnóstico da doença atual } \\
\hline \multicolumn{5}{|c|}{ Colecistite aguda } \\
\hline Sim & 2,86 & $0,58-14,09$ & 6,84 & $0,00-0,00$ \\
\hline Não & 1,00 & & 1,00 & \\
\hline \multicolumn{5}{|l|}{ Colelitíase } \\
\hline Sim & 0,00 & $0,00-0,00$ & 1,96 & $0,00-0,00$ \\
\hline Não & 1,00 & & 1,00 & \\
\hline \multicolumn{5}{|c|}{$\begin{array}{l}\text { Tempo de internação até a alta } \\
\text { hospitalar }\end{array}$} \\
\hline Média & 1,08 & $0,99-1,18$ & 1,10 & $1,00-1,22$ \\
\hline Desvio Padrão & 1,00 & & 1,00 & \\
\hline
\end{tabular}

*RC: Razões de Chances; †IC 95\%: Intervalo de Confiança de 95\%; Análise Ajustada: Todas as variáveis foram introduzidas no modelo ajustado independentemente do valor de $p$. Permaneceram no modelo ajustado as variáveis com $p \leq 0,200$

Pela análise de regressão logística binária, os fatores de risco do pós-operatório hospitalar influenciáveis no surgimento das ISC em domicílio, tanto na análise ajustada quanto na bruta não tiveram critérios de significância e risco, já que o evidenciado e estatisticamente pelo intervalo de IC 95\% é acima de 1.

Tabela 4 - Análise de regressão logística binária dos fatores de risco do período pós-operatório na presença de infecções do sitio cirúrgico no pós-operatório domiciliar. Florianópolis, SC, Brasil, 2015

\begin{tabular}{|c|c|c|c|c|}
\hline \multirow{2}{*}{ Fatores de risco } & \multicolumn{2}{|c|}{ Análise bruta } & \multicolumn{2}{|c|}{ Análise ajustada } \\
\hline & $\mathrm{RC}^{*}$ & IC 95\%† & $\mathrm{RC}^{*}$ & IC 95\%† \\
\hline \multicolumn{5}{|l|}{ Tipo de cirurgia } \\
\hline \multicolumn{5}{|l|}{ Colecistectomia } \\
\hline Sim & 0,47 & $0,18-1,22$ & 0,38 & $0,14-1,05$ \\
\hline Não & 1,00 & & 1,00 & \\
\hline \multicolumn{5}{|l|}{ Oxigenioterapia } \\
\hline Sim & 0,30 & $0,07-1,19$ & 0,24 & $0,05-1,06$ \\
\hline Não & 1,00 & & 1,00 & \\
\hline \multicolumn{5}{|l|}{$\begin{array}{l}\text { Tempo de internação até a } \\
\text { alta hospitalar }\end{array}$} \\
\hline Média & 0,97 & $0,91-1,03$ & 0,98 & $0,91-1,04$ \\
\hline Desvio Padrão & 1,00 & & 1,00 & \\
\hline
\end{tabular}

*RC: Razões de Chances; †IC 95\%: Intervalo de Confiança de 95\%; Análise ajustada: Todas as variáveis foram introduzidas no modelo ajustado independente do valor de $p$. Permaneceram no modelo ajustado as variáveis $\operatorname{com} p \leq 0,200$ 


\section{DISCUSSÃO}

No presente estudo, os casos constatados com ISC, foram classificados e comprovados conforme a elaboração de alguns estudos na área. 8,18-22

A prevalência de ISC no hospital e no domicílio do estudo foi de sete $(8,3 \%)$ e $41(48,8 \%)$, respectivamente. Este resultado é considerado aceitável e coincide com outros estudos, como o realizado na Amazônia que encontrou o total de 81 (3,68\%) ao analisar 2203 pacientes no período de 2009 a 2010. Destes, 59 (72,844\%) ocorreram em cirurgias potencialmente contaminadas, e no presente estudo todas as cirurgias tiveram esta classificação, corroborando com o potencial de contaminação aceito pelo Center for Desease Control and Prevention (CDC) que preconiza que 3 a $11 \%$ ocorrem em cirurgias potencialmente contaminadas. Em outra pesquisa, foi constatada uma incidência de 115 (3,70\%) ao analisar 3120 participantes no período de 1999 a $2002{ }^{23-29}$

Os fatores de risco relacionados ao desenvolvimento da ISC no período pós-operatório hospitalar que se destacaram nesta pesquisa foram as cirurgias de colecistectomia, as doenças de colecistite aguda e colelitíase e o período de internação hospitalar até a alta, dentre os quais corroboram com as pesquisas científicas. ${ }^{8,21,25-26}$ Porém a colecistectomia e o diagnóstico de colecistite aguda não foram elementos significativos e diferenciais para certificar que os pacientes que apresentaram estes fatores, sofreram mais ISC hospitalar do que aqueles que não foram submetidos a esta intervenção cirúrgica, tampouco diagnosticados com a doença ${ }^{33}$ esses resultados não comprometeram o alcance dos objetivos propostos por esta pesquisa estatisticamente.

Nos países ocidentais, a presença das ISC hospitalares corresponde em média de $15 \%$ a $20 \%$ de todos os casos de infecções voltadas para a assistência à saúde. Esta incidência acomete dois a 15 casos de infeção para cada 100 pacientes submetidos à cirurgia geral. A OMS lançou no ano de 2009 um desafio global para a segurança do paciente que buscou minimizar as chances e os riscos ao desenvolvimento das ISC. Em uma pesquisa retrospectiva, analisou-se os dados de 2008 à 2009 de 247 pacientes submetidos ao procedimento de colecistectomia e revelou que em dois $(0,8 \%)$ casos houve o surgimento da ISC hospitalar. ${ }^{31,34}$

A colecistectomia é um procedimento de alta demanda assistencial que consiste na retirada da vesícula biliar e é considerada a cirurgia potencialmente contaminada mais realizada no mundo, ficando com a segunda colocação no ranking das cirurgias abdominais. É um procedimento indicado no tratamento da colelitíase e suas complicações, como a colecisitite aguda e em casos de neoplasias da vesícula biliar. Aproximadamente 500.000 casos destes procedimentos são realizados anualmente nos Estados Unidos. Colecistolitíase é uma das doenças mais comuns do aparelho digestório, e constitui um importante problema de saúde nos países desenvolvidos. Estima-se que $10 \%$ a $15 \%$ da população adulta, representando 20 a 25 milhões de americanos, têm ou terão cálculos biliares. O Brasil apresenta prevalência de 9,3\% de casos de colelitíase na população em geral, demandando cerca de 60.000 internações por ano no Sistema Único de Saúde (SUS). ${ }^{21-22,35-46}$

Em outro estudo ${ }^{23}$ foram investigados 2.203 participantes submetidos a procedimentos anestésico-cirúrgicos, 81 (3,68\%) apresentaram ISC, sendo que deste total $59(72,84 \%)$ foram de cirurgias potencialmente contaminadas, ainda que a cirurgia de colecistectomia foi a mais destacada, com 38 $(46,91 \%)$ dos casos investigados.

Na doença da vesícula biliar aguda não complicada a evidência atual sugere que colecistectomia laparoscópica precoce é segura e diminui o período de internação. ${ }^{24}$

Em uma pesquisa ${ }^{27}$ realizada com 428 sujeitos entre os anos de 2008 e 2009, a associação da colecistite com as ISC hospitalares foi identificada em 21 casos de ISC de cirurgias potencialmente contaminadas (colecistectomia videolaparoscópica). Todos os participantes apresentaram, pelo exame de anatomopatológico, o diagnóstico de colecistite calculosa.

Com relação a colelitíase, a mesma é considerada a doença cirúrgica abdominal mais comum no paciente idoso, sendo sua incidência relacionada à progressão da idade, com prevalência global na população em geral, de 9,3\%. ${ }^{39}$ A colelitíase é um dos principais sintomas relacionados ao surgimento da colecistite aguda. Assim como na literatura, esse achado não foi representativo para o desenvolvimento da ISC hospitalar. ${ }^{18,35-36,46-48}$

Sobre o tempo de internação hospitalar, quanto menor este período, menores são as probabilidades de um paciente desenvolver uma infecção associada a assistência à saúde. Retratado ao paciente cirúrgico, esse momento tem sido cada vez menor, normalmente em torno do segundo ou terceiro dia de pós-operatório e por isso o acompanhamento após a alta hospitalar é fundamental, já que as ISC podem ocorrer em até 30 dias após a realização da cirurgia. Nos estudos investigados, constatou-se 
que o tempo de internação hospitalar até a alta, é considerado fator predisponente ao surgimento da ISC, visto que estas infecções levam a um aumento médio de $60 \%$ no tempo de internação. ${ }^{32,40-41}$

A maioria dos pacientes ficou internado até um dia de pós-operatório hospitalar (71, correspondendo a $84,52 \%$ do total), $10(11,90 \%)$ em dois dias e $3(3,57 \%)$ por três dias após procedimento cirúrgico. ${ }^{33}$

Em uma pesquisa, o total de dias de internação, contados entre a baixa e a alta hospitalar (dias de permanência) foi maior entre os 50 a 59 anos ( $21,3 \%$ do total). Já a maior média de permanência ocorreu na faixa etária de 0 a 4 anos (nove dias), seguida dos pacientes com 80 anos ou mais (sete dias), acima do verificado para o estado do Rio Grande do Sul, de quatro dias de permanência. Ao se analisar a taxa de incidência de ISC de acordo com o tempo de permanência hospitalar pré-cirúrgico, pudemos observar uma elevação crescente da taxa de ISC de acordo com o tempo da permanência. Quanto a taxa de incidência de ISC de acordo com o tempo de permanência hospitalar pré-cirúrgico, observou-se uma elevação crescente da taxa de ISC de acordo com o tempo da permanência. Mesmo diante desse fato, a variável tempo de hospitalização pré-cirúrgico não se mostrou significante, no corrente estudo (p-valor $=0,1420) \cdot{ }^{26-27}$

A média do tempo de internação hospitalar para os pacientes que desenvolveram ISC no ambiente hospitalar, foi de 13,71 dias, já para aqueles que tiveram ISC em domicílio, este período foi de 8,51 dias. Refletindo sobre esse achado, alguns estudos apresentam este momento maior do que o comprovado pela pesquisa que determinou uma média de 49,2 dias para aqueles que evoluíram com ISC. ${ }^{12,16}$

Na ISC o tempo que corresponde ao período de incubação é de três a oito dias após a cirurgia, sendo este momento determinado pelas variáveis de classificação da ferida. Tendo em vista as alterações que ocorrem no pós-operatório, é fundamental um acompanhamento ao paciente para que ele retorne as suas atividades diárias mais rapidamente, reduzindo os risco e chances para o desenvolvimento das infecções no ambiente hospitalar. ${ }^{46}$

Diante do aspecto epidemiológico, as ISC adquirem grande importância por contabilizarem de 20 a 31\% das infecções entre pacientes hospitalizados. Além do mais é a infecção que produz mais mortalidades, complicações e elevações nos custos do tratamento de pacientes cirúrgicos. O impacto clínico desse fato implica no aumento da permanên- cia hospitalar em 3 a 15 dias extras de internação, quando comparados a paciente que adquirem ISC com aqueles sem ISC. ${ }^{47}$

Com relação ao fator de risco o uso de oxigenioterapia, no presente estudo não houve comprovação estatística de que esta variável teria relação com o desencadeamento das ISC no período pós-operatório domiciliar. Nas literaturas investigadas, também não houve associação significativa deste fator de risco com o surgimento dessa infecção. Porém, dentro das infecções hospitalares, a administração de oxigenioterapia é fator predisponente ao desenvolvimento das infecções respiratórias. ${ }^{2,14,23,35,48-49}$

Ainda que neste estudo o período de internação hospitalar não tenha sido estatisticamente comprovado como fator de risco para o desenvolvimento de ISC tanto no domicílio, como no hospitalar, existe evidência de que o controle no pós-alta do paciente cirúrgico fundamenta os reais índices da ISC. Muitos deles, confirmam que por meio do seguimento pós-alta são constatadas diversas que confirmam a necessidade deste tipo de vigilância. A maioria dos pacientes cirúrgicos, principalmente nos países mais pobres, recebe alta precoce, por conta da falta de leitos e ao alto custo de tratamentos. Por isto as ISC obtidas somente em pacientes internados, não refletem o real desenvolvimento desta infecção, principalmente nos procedimentos em que o tempo de permanência pós-operatório é menor. ${ }^{22,50-53}$

Sendo a ISC a mais importante causa das complicações pós-operatória do paciente cirúrgico, para o seu reconhecimento é necessário o estabelecimento de estratégias como a vigilância pós-alta, considerando que 12 a $84 \%$ das ISC são diagnosticadas durante este monitoramento. Esta investigação é crucial para a obtenção de indicadores no intuito de diminuir a subnotificação destas infecções e consequentemente a subestimação da real incidência, impacto e relevância da ISC. A taxa de incidência de ISC encontrada neste estudo foi de 8,7\%. A maioria dos casos $(61,9 \%)$ foi diagnosticada após a alta hospitalar, o que sugere a importância de um serviço de vigilância pós-alta especializado. ${ }^{22,30,32,50}$

No Brasil, a maioria das instituições limita-se a vigilância do surgimento da ISC durante a internação hospitalar e não abrange o acompanhamento após alta. O CDC recomenda que os pacientes cirúrgicos recebam controle e vigilância ampliada até o período pós-operatório domiciliar. Isso faz com que a responsabilidade em manter o paciente seguro não seja só dos profissionais que prestam assistência, mas de todos os componentes do sistema - gerentes, administradores e instituições em geral. ${ }^{22,54-55}$ 
Os métodos de seguimento do paciente após a alta mais utilizados podem ser: busca ativa, notificação passiva, revisão de prontuários, avaliação de exames, revisão de banco de dados. Além disso, é importante segregar a interface com a Estratégia de Saúde da Família (ESF), no intuito de fortalecer a referência e contra referência e capacitações de todos os profissionais que atuarão na assistência básica ao paciente cirúrgico em pós-operatório. ${ }^{47}$

Estudos também revelam que a criação de comissões de segurança do paciente, identificação do paciente, protocolos, dupla checagem, incentivo ao relato de erros e eventos adversos são incentivadas pelo programa de segurança do paciente, demonstrando assim a sensibilização quanto a importância. ${ }^{56}$

\section{CONCLUSÕES}

Os resultados apontam que não houve diferença significativa nos fatores de risco apresentados com o desenvolvimento das ISC a nível hospitalar e domiciliar, como resultado, estes fatores foram coletados e investigados, pois em diversos estudos foram comprovados e associados às infecções. Isto se deu pelo fato de que algumas limitações ocorreram como período de coleta de dados em curto prazo, inviabilidade no acompanhamento diário quando ocorrido intercorrências com os participantes em pós-operatório.

Os Serviços de Controle de Infecção Hospitalar necessitam atuar no monitoramento aos pacientes cirúrgicos, no intuito de reduzir as chances e prevalência da ISC, principalmente por conta dos altos níveis de procedimentos cirúrgicos, ficando assim os pacientes em pós-operatório expostos em maior escala, já que quanto mais o número de intervenções cirúrgicas, menor o tempo de internação hospitalar, devido o número de leitos reduzidos. O serviço de referência e contra referência pode aprimorar e qualificar a assistência ao paciente cirúrgico, através do desenvolvimento de uma rede de cuidados com a equipe da ESF e profissionais de saúde de unidades hospitalares. Ainda assim fica a sugestão para que as instituições implantem um serviço de vigilância e acompanhamento pós-alta, a fim de melhorar a confiabilidade das taxas de incidência das ISC, melhorar a qualidade dos cuidados com os pacientes, compreensão epidemiológica e principalmente, identificar os fatores de risco predisponentes das ISC, viabilizando a implementação de soluções e medidas direcionadas a sua prevenção e controle.

\section{REFERÊNCIAS}

1. Cheregatti, AL. Enfermagem em clínica cirúrgica no pré e no pós operatório. $1^{\mathrm{a}}$ ed. São Paulo (SP): Editora Martinari; 2012.

2. Agência Nacional de Vigilância Sanitária. Critérios diagnósticos de infecção relacionada à assistência à saúde. Série segurança do paciente e qualidade em serviços de saúde. $2^{\mathrm{a}}$ ed. Brasília (DF): ANVISA; 2017.

3. Sasaki VDM, Romanzini AE, Jesus APM, Carvalho E, Gomes JJ, Damiano VB. Vigilância de infecção de sítio cirúrgico no pós-alta hospitalar de cirurgia cardíaca reconstrutora. Texto Contexto Enferm [Internet]. 2011 [cited 2017 Mar 22]; 20(2):328-32. Available from: http://www.scielo.br/scielo.php?script=sci_arttext \&pid=S0104-07072011000200015

4. Monteiro TS, Pedroza RM. Infecção hospitalar: visão dos profissionais da equipe de enfermagem. Rev Epidemiol Control Infect. 2015;5(2):84-8

5. Morales CLP, Alexandre JG, Prim S, Amante LZ. Perioperative communication from the perspective of patients undergoing bariatric surgery. Text Context Nursing [internet]. 2014 [cited 2017 Mar 22]; 23(2):347-55. Available from: http:// www.scielo.br/scielo.php?script=sci_arttext\&pid =S0104-07072014000200347

6. Bass JB Jr, Farer LS, Hopewell PC, O'Brien R, Jacobs RF, Ruben F, et al. Treatment of tuberculosis and tuberculosis infection in adults and children. American Thoracic Society and The Centers for Disease Control and Prevention. Am J Respir Crit Care Med [internet]. 1994 May [cited 2017 Mar 22];149(5):1359-74. Available from: https://www. ncbi.nlm.nih.gov/pubmed/8173779

7. Maya MAS, Interacciones en el cuidado de enfermería al paciente en la etapa perioperatoria. Rev Cub Enferm. 2012; 28(4):495-508.

8. Batista TF, Rodrigues MCS. Vigilância de infecção do sítio cirúrgico pós-alta hospitalar em hospital de ensino do Distrito Federal, Brasil: estudo descritivo retrospectivo no período 2005-2010. Epidemiol. Serv Saúde [Internet] 2012 [cited 2015 Oct 22]; 21(2):253-64. Available from: http://scielo.iec.pa.gov.br/pdf/ess/ v21n2/v21n2a08.pdf.

9. Oliveira EM, Paula JB. Fatores associados à infecção de sítio cirúrgico em pacientes idosos submetidos à cirurgia cardíaca com esternotomia. Saúde Santa Maria. 2014; 40(1):37-44.

10. Feitosa RGF, Fernandes FAM, Narciso J Jr, Araújo ON Jr, Costa FA, Cavalcante LDW. Análise da incidência de infecção do sítio cirúrgico em cirurgias oncológicas do aparelho digestivo no Hospital Geral de Fortaleza. Medicina (Ribeirão Preto). 2014; 47(2):157-64

11. Oliveira AC, Gama CS. Evaluation of adherence to measures for the prevention of surgical site infections by the surgical team. Rev Esc Enferm USP. 2015 [cited 2017 Mar 22]; 49(5):764-70. Available from: http:// 
www.scielo.br/scielo.php?script=sci_arttext\&pid $=$ S0080-62342015000500767

12. Feitosa RGF, Fernandes FAM, Júnior JN, Júnior ONA, Costa FA, Cavalcante LDW. Análise da incidência de infecção do sítio cirúrgico em cirurgias oncológicas e do aparelho digestivo no Hospital Geral de Fortaleza. Medicina (Ribeirão Preto) [Internet] 2014 [cited 2015 Oct 22]; 47(2):157-64. Available from: http:// revista.fmrp.usp.br/2014/vol47n2/AO3_Analiseda-incidencia-de-infeccao-de-sitio-cirurgico-emcirurgias-oncologicas-do-aparelho-digestivo.pdf

13. Castro PMV, Akerman D, Munhoz CB, Sacramento I, Mazzurana M, Alvarez GA. Laparoscopic cholecystectomy versus minilaparotomy in cholelithiasis: systematic review and meta-analysis. ABCD Arq Bras Cir Dig. 2014; 27(2):148-53.

14. Santana CA, Oliveira CGE. Assistência de enfermagem na prevenção de infecções de sítio cirúrgico: uma revisão integrativa da literatura. Rev Eletrôn Atualiza Saúde [Internet]. 2015 [cited 2017 Mar 22]; 1(1): Available from: http://atualizarevista.com. br/wp-content/uploads/2015/01/ASSISTENCIADE-ENFERMAGEM - N A - PREVENCAODE-INFECCOES-DE-SITIO-CIRURGICOUMA-REVIS\% C3\% 83O-INTEGRATIVA-DALITERATURA-REVISTA-ATUALIZA-SAUDEN1-V1.pdf

15. Masukawa II, Vieira GB, Klein TR. Serviços de Controle de Infecção Hospitalar (SCIH/HU). Bol Epidemiol. 2014.

16. Santos GC, Baylão AFG, Borges SCF, Silva LA, Batista MHJ, Leite GR. Incidência e fatores de risco de infecção do sítio cirúrgico: uma revisão integrativa. Rev Eletr Itinerarius Reflectionis [Internet]. 2015 [cited 2015 Oct 22]; 11(1). Available from: https:/ / revistas. ufg.emnuvens.com.br/ritref/article/view/34142.

17. Nassar SM. SEstatNET- Sistema Especialista para o Ensino de Estatística na Web [Internet]. 2011 [cited 2017 Mar 22]. Available from: http://www.sestatnet.ufsc.br

18. Aragão JMN. Infecção do sítio cirúrgico em pacientes submetidos à colecistectomia convencional e videolaparoscópica em Hospital Regional do Distrito Federal: identificação do momento de diagnóstico e incidência [monografia Internet]. Brasília (DF): Universidade de Brasília, Faculdade de Ceilândia; 2013 [cited 2015 Nov 02]. Available from: http://bdm.unb.br/bitstream/10483/5932/1/2013_ JulisseMarcelaNepomucenoAragao.pdf $>$

19. Laura M, Shaffer S, Shaffer EA. Epidemiology of Gallbladder Disease: Cholelithiasis and Cancer. Gut Liver [Internet]. 2012 [cited 2017 Mar 22]; 6(2):17287. Available from: https:// www.ncbi.nlm.nih.gov/ pmc/articles/PMC3343155/pdf/gnl-6-172.pdf

20. Castro PMV, Akerman D, Munhoz CB, Sacramento I do, Mazzurana M, Alvarez GA. Colecistectomia laparoscópica versus minilaparotômica na colelitíase: revisão sistemática e metanálise. ABCD Arq Bras Ci.
Dig. 2014 Abr-Jun [cited 2017 Mar 22]; 27(2):148-53. Available from: http://www.scielo.br/pdf/abcd/ v27n2/pt_0102-6720-abcd-27-02-00148.pdf

21. Lemos FN, Oliveira AV, Sousa MG. Infecção do Sítio Cirúrgico: estudo prospectivo de 2.149 pacientes operados. Rev Colégio Brasileiro de Cirurgiões [Internet] 1999 [cited 2015 Nov 02]; 26(2):109-13. Available from: http://www.scielo.br/pdf/rcbc/ v26n2/07.pdf

22. World Health Organization. Global guidelines for the prevention of surgical siteinfection. Geneva:WHO;2016 [cited 2017 Mar 22]. Available from: http:/ / apps.who. int/iris/bitstream/10665/250680/1/9789241549882eng.pdf?ua $=1$

23. Aguiar APL, Prado PR, Opitz SP, Vasconcelos SP, Faro ARMC. Fatores associados à infecção de sítio cirúrgico em um hospital na Amazônia ocidental brasileira. Rev SOBECC [Internet] 2012 [cited 2015 Oct 22]; 17(3):60-70. Available from: https:/ / revista. sobecc.org.br/sobecc/article/view/168

24. Sankarankutty A, Luz LT, Campos T, Rizoli S, Fraga GP, Nascimento Jr B. Colecistite aguda nãocomplicada: colecistectomia laparoscópica precoce ou tardia?. Rev Col Bras Cir [Internet]. 2012 Oct [cited 2017 Feb 23]; 39(5):436-40. Available from: http://www. scielo.br/scielo.php?script=sci_arttext\&pid=S010069912012000500017\&lng=en

25. Medeiros AC, Neto TA, Filho AMD, Júnior FELP, Uchôa RAC, Carvalho MR Infecção hospitalar em pacientes cirúrgicos de Hospital Universitário. Acta Cir Bras [Internet] 2003 [cited 2015 Nov 02]; 18(1):15-8. Available from: http://www. scielo.br/scielo.php?script=sci_arttext\&pid=S0102$86502003000700003 \& \operatorname{lng}=$ en\&nrm $=$ iso\&tlng $=p t$

26. Nunes EC, Rosa RS, Bordin R. Internações por colecistite e colelitíase no Rio Grande do Sul, Brasil. ABCD Arq Bras Cir Dig 2016 [cited 2017 Mar 22]; 29(2):77-80. Available from: http://www.scielo.br/ pdf/abcd/v29n2/pt_0102-6720-abcd-29-02-00077.pdf

27. Rodrigues ALS, Miranda AC, Dourado CJC, Almeida DPR, Brito NB, Araújo RS. Avaliação de pacientes quanto à infecção de sítio cirúrgico, em um hospital público de BELÉM-PA. Rev Paraense Med [Internet] 2014 [cited 2017 Mar 22]; 28(1):. Available from: http:/ / files.bvs.br/upload/S/0101-5907/2014/ v28n1/a4160.pdf

28. Culver DH, Horan TC, Gaynes RP, Martone WJ, Jarvis WR, Emori TG, et al. Surgical wound infection rates by wound class operative procedure, and risk index. Am J Med. [Internet] 1991 [cited 2015 Nov 02]; 91(3):152-7. Available from: http://www.ncbi.nlm. nih.gov/pubmed/1656747

29. Sociedade Brasileira de Enfermeiros de Centro Cirúrgico, Recuperação Anestésica e Centro de Material e Esterilização (SOBECC). Práticas recomendadas SOBECC. $5^{\text {a }}$ ed: São Paulo (SP): SOBECC; 2013. 
30. Rodrigues ALS, Miranda AC, Dourado CJC, Almeida DPR, Brito NB, Araújo RS. Avaliação de pacientes quanto à infecção de sítio cirúrgico, em um hospital público de BELÉM-PA. Rev Paraense Med [Internet] 2014 [cited 2017 Mar 22]; 28(1):. Available from: http:/ / files.bvs.br/upload/S/0101-5907/2014/ v28n1/a4160.pdf

31. MeloEMVB, Leão CS, Andreto LM, Mello MJG. Infecção cirúrgica em colecistectomia videolaparoscópica usando ácido peracético como esterilizante dos instrumentais. Rev Col Bras Cir [Internet] 2012 [cited 2015 Oct 28]; 40(3):208-14. Available from: http:// www.scielo.br/pdf/rcbc/v40n3/08.pdf

32. Oliveira AC, Ciosak SI. Infecção do sítio cirúrgico em hospital universitário: vigilância pós-alta e fatores de risco. Rev Esc Enferm USP [Internet] 2007 [cited 2015 Oct 10]; 41(2):258-63. Available from: http://www. ee.usp.br/reeusp/upload/pdf/711.pdf

33. Senne ECV. Avaliação de prevalência e fatores associados à infecção do sítio cirúrgico em colecistectomia videolaparoscópica antes e após a implantação da vigilância pós-alta (dissertação). Uberaba (MG): Universidade Federal do Triângulo Mineiro, Curso de Pós Graduação em patologia [Internet] 2011 [cited 2015 Oct 29]. Available from: http://sitioanterior.uftm.edu.br/patolo/cpgp/ imagem/Tese_EvaCVSenneME.pdf.

34. Ferreira FAPB, Marin MLG, Strabelli TMV, Carmona MJC. Como o anestesiologista pode contribuir para a prevenção da infecção no paciente cirúrgico. Rev Bras Anestesiol. [Internet] 2009 [cited 2015 Oct 29]; 59(6):756-66. Available from: http:// www.scielo.br/scielo.php?script=sci_arttext\&pid =S0034-70942009000600013

35. Santos JSS, Sankarankutty AK, Júnior WS, Kemp R, Módena JLP, Júnior JE. Colecistectomia: aspectos ténicos e indicações para o tratamento da litíase biliar e das neoplasias. Medicina (Ribeirão Preto) [Internet] 2008 [cited 2015 Nov 02]; 41(4):449-64. Available from: http:/ / revista.fmrp.usp.br/2008/VOL41N4/ SIMP_3colecistectomia.pdf

36. Oliveira AC, Garcia CA, Scatena PD, Soares JL, D'Osvaldo L, Ciosak SI, et al. Incidência de infecção do sítio cirúrgico (isc) em pacientes submetidos à colecistectomia por duas técnicas: convencional e videolaparoscópica. Rev Min Enferm [Internet] 2004 [cited 2015 Nov 02]; 8(1):191-5. Available from: http:/ / www.reme.org.br/artigo/detalhes/752.

37. Coelho JCU, Campos ACL. Litíase vesicular e colecistite. In: Kalil NA, Coelho J, Strauss E. Fígado e vias biliares clínica e cirurgia. Rio de Janeiro (RJ): Editora Revinter; 2001.

38. Salim MT, Cutait R. Complicações da cirurgia videolaparoscópica no tratamento de doenças da vesícula e vias biliares. Arq Bras Cir Dig [Internet] 2008 [cited 2015 Oct 29]; 21(4):153-7. Available from: http:/ / www.scielo.br/scielo.php?pid=S010267202008000400001\&script=sci_arttext
39. Olsen DO. Laparoscopic cholecystectomy. Am J Surg [Internet] 1991 [cited 2015 Oct 23]; 161(3):339-44. Available from: http:// www.americanjournalof surgery.com / article/0002-9610\%2891\%2990592-2/abstract

40. Goffi FS, Júnior PSG, Sorbello AA. Cirurgia das vias biliares. In: Goffi FS, Schmidt F. Bases anatômicas, fisiopatológicas e técnicas da cirurgia. $4^{\text {a }}$ ed. Rio de Janeiro (RJ): Editora Atheneu; 2001.

41. Freitas ML, Bell RL, Duffy AJ. Choledocholithiasis: evolving standars for diagnosis and management. World J Gastroenterol [Internet] 2006 [cited 2015 Oct 29]; 12(20):3162-7. Available from: http:/ / europepmc. org/articles/pmc4087957

42. Schirmer BD, Winters KL, Edlich R. Cholelithiasis and cholecystitis. J. Long Term Eff Med Implants [Internet]. 2005 [cited 2015 Oct 29]; 15(3):329-38. Available from: http:/ / www.dl.begellhouse.com/ journals/1bef42082d7a0fdf,69622d0e4ea6cf4b,07f88f 8255346b09.html

43. Nakeeb A, Comuzzie AG, Martin L, Sonnenberg GE, Schwartz-Basile D, Kissebah AH, et al. Gallstone: genetics versus environment. Ann Surg [Internet]. 2002 [cited 2015 Oct 29]; 235(6):842-9. Available from: http://www.ncbi.nlm.nih.gov/pmc/articles/ PMC1422514/

44. Maya MCA, Freitas RG, Pitombo MB, Ronay A. Colecistite aguda: diagnóstico e tratamento. Rev Hospital Universitário Pedro Ernesto [Internet]. 2009 [cited 2015 Oct 29]; 8(1):52-60. Available from: http:/ / revista.hupe.uerj.br/detalhe_artigo.asp?id=169

45. Altenfelder SR. Complicações da colecistectomia vídeo laparoscópica. In: Margarido NF. Videocirurgia. São Paulo (SP): Editora Robe; 1994.

46. Saltiél RV, Pedrini A, Paulin E. Perfil dos pacientes submetidos à colecistectomia no Hospital Regional de São José Dr. Homero de Miranda Gomes na grande Florianópolis-SC. Arq Cienc Saúde UNIPAR [Internet]. 2011 [cited 2015 Nov 01]; 15(2):173-9. Available from: http://revistas.unipar.br/index. $\mathrm{php} /$ saude/article/view/3713

47. Oliveira AC, Silva MVG. Teoria e prática na prevenção da infecção do sítio cirúrgico. Barueri (SP): Editora Manole; 2015.

48. Gebrim CFL, Melchior LMR, Amaral NM, Barreto RASS, Palos MAP. Tricotomia pré-operatória: aspectos relacionados à segurança do paciente. Enfermería Global [Internet] 2014 [cited 2015 Nov 01]; 34: 264-75. Available from: http://scielo.isciii.es/pdf/ eg/v13n34/pt_administracion3.pdf.

49. Machado A, Ferraz AAB, Ferraz E, Arruda E, Nobre J, Konkewicz LR, et al. Prevenção da Infecção Hospitalar. Projeto Diretrizes [Internet] 2001 [cited 2015 Nov 01]. Available from: http://www.projetodiretrizes.org. br/projeto_diretrizes/065.pdf.

50. Burns SJ, Dippe SE. Postoperative wound infections detected during hospitalization and after discharge in 
a community hospital. Am J Infect Control [Internet]. 1982 [cited 2015 Nov 02]; 10(2):60-5. Available from: http:// www.sciencedirect.com/science/article/ pii/0196655382900049

51. Froggatt JW, Mayhall CG. Development and validation of a surveillance system for postoperative wound infections in a university center. In: Annual Meeting of the American Society for Microbiology. New Orleans, 1989. p. 14-8;

52. Grinbaum RS. Infecções do sítio cirúrgico e antibioticoprofilaxia em cirurgia. In: Rodrigues EAC, Mendonça JS, Amarante JMB, Filho MBA, Grinbaum RS, Richtmann Rr. Infecções hospitalares: prevenção e controle. São Paulo (SP): Editora Sarvier; 1997.

53. Oliveira AC. Controle de egresso cirúrgico: impacto na incidência da infecção do sítio cirúrgico em Hospital Universitário [dissertação]. Belo Horizonte (MG): Universidade Federal de Minas Gerais, Escola de Enfermagem; 1999.
54. Oliveira AC, Carvalho DV. Avaliação da subnotificação da infecção do sítio cirúrgico evidenciada pela vigilância pós-alta. Rev Latino-Am Enfermagem [Internet] 2007 [cited 2015 Nov 01]; 15(5):117-22. Available from: http://www.scielo.br/scielo. php?pid $=$ s0104-11692007000500017\&script $=$ sci $_{-}$ arttext\&tlng=pt

55. Silva LD. Segurança do paciente no contexto hospitalar. Rev Enferm UERJ [Internet] 2012 [cited 2012 Jul]; 20(3):291-2. Available from: http:/ / www.epublicacoes.uerj.br/index.php/enfermagemuerj/ article/view/4076

56. Mello JF, Barbosa SFF. Cultura de segurança do paciente em terapia intensiva: recomendações da enfermagem. Texto Contexto Enferm [Internet] 2013 [cited 2017 Mar 22]; 22(4):1124-33. Available from: http://www.scielo.br/pdf/tce/v22n4/31.pdf. 